\title{
COMPARAÇÃO ENTRE EQUAÇÕES DE REFERÊNCIA E O TESTE DE CAMINHADA DE SEIS MINUTOS
}

\author{
COMPARATION BETWEEN REFERENCE EQUATIONS AND THE SIX-MINUTE WALK TEST \\ COMPARACIÓN DE ECUACIONES DE REFERENCIA Y PRUEBA DE CAMINATA DE SEIS MINUTOS
}

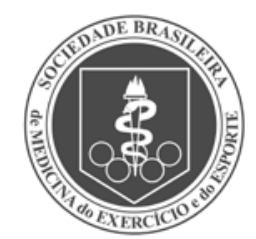

Artigo Original
Simone Korn ${ }^{1}$

(Educadora Física e Fisioterapeuta)

Janeisa Franck Virtuoso ${ }^{2}$

(Fisioterapeuta)

Paula Fabricio Sandreschi ${ }^{2}$

(Graduanda de Educação Física)

Michelle Gonçalves de Souza ${ }^{3}$

(Fisioterapeuta)

Giovana Zarpellon Mazo²

(Educadora Física)

1. Universidade do Sul de Santa Catarina, UNISUL, Palhoça, SC, Brasil.

2. Universidade do Estado de Santa Catarina, UDESC,

Florianópolis, SC, Brasil.

3. Universidade Federal de Santa

Catarina, UFSC, Florianópolis,

SC, Brasil.

\section{Correspondência:}

Giovana Zarpellon Mazo

Universidade do Estado de Santa

Catarina - UDESC - Florianópolis/SC.

Rua Pacoal Simone, 358, Coqueiros,

Florianópolis, SC, Brasil. 88080-350

giovana.mazo@udesc.br

\section{RESUMO}

Introdução: Devido à importância do teste de caminhada de seis minutos (TC6), algumas equações têm sido propostas para predizer o resultado esperado. Objetivo: Comparar os valores encontrados em diferentes equações de referência para predição da distância percorrida no TC6 em idosos praticantes de exercícios físicos. Métodos: Participaram 696 idosos de ambos os sexos, sendo 600 mulheres e 96 homens, praticantes de exercícios físicos do Grupo de Estudos da Terceira Idade (GETI), Programa Viver Ativo e do Programa Saúde e Lazer. Para avaliar a resistência aeróbia, foi utilizado o TC6, conforme proposto por Rikli e Jones. Para o cálculo da distância percorrida prevista, foram utilizadas as equações de Enright \& Sherrill, Troosters e Enright. Utilizou-se estatística descritiva e inferencial, com nível de significância de 5\%. Resultados: A média

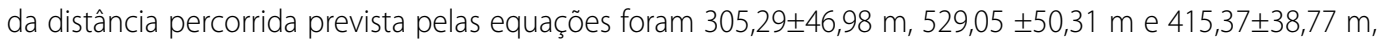
respectivamente. No TC6, a média foi de 513,05 $\pm 87,26$ m. Observou-se correlação significativa $(p<0,001)$ entre o TC6 e as equações de Enright \& Sherrill e Troosters. Na comparação entre os valores, nota-se que as equações de Enright \& Sherrill e Enright subestimam e as de Troosters superestima a resistência aeróbia de idosos praticantes de exercícios físicos. Conclusão: As equações de referência para predição da distância percorrida no TC6 analisadas no presente estudo não refletem a resistência aeróbia de idosos praticantes de exercícios físicos. Novas equações e/ou variáveis devem ser incluídas para calcular com certa precisão a distância percorrida por idosos fisicamente ativos. Dessa forma, as equações analisadas no presente estudo devem ser utilizadas com cautela por pesquisadores e profissionais que lidam com este público no Brasil.

Palavras-chave: atividade física, exercício, resistência física, envelhecimento.

\section{ABSTRACT}

Introduction: Due to the importance of the six minutes walking test (6MWT), some equations have been proposed to predict the expected results. Objective: To compare the values found in different reference equations to predict the distance covered on the 6MWT in the elderly that do physical exercises. Methods: The study included 696 elderly of both sexes, 600 women and 96 men, who do physical exercises of the Study Group of the Elderly (GETI), Active Living Program and the Health and Recreation Program. To assess aerobic resistance, the 6MWT was used, as proposed by Rikli and Jones. To calculate the covered distance, the equations of Enright \& Sherrill, Troosters e Enright were used. We used the descriptive and inferential statistics, with significance level of $5 \%$. Results: The mean covered distance predicted by the equations was 305.29 ( \pm 46.98$), 529.05$ ( \pm 50.31$)$ and $415.37( \pm 38.77)$ meters, respectively. In the 6MWT, the mean was 513.05 ( \pm 87.26$)$ meters. A significant correlation $(p<0.001)$ between the 6MWT and the equation of Enright \& Sherrill and Troosters was observed. When comparing the values, we can notice that the equations of Eringht \& Sherrill and Enright underestimate aerobic endurance of elderly individuals that do exercises and the Troosters equation overestimates these individuals. Conclusion: The reference equations that predict the covered distance in 6MWT analyzed in the current study do not reflect the aerobic resistance of elderly people who do physical exercises. New equations and/or variables must be included to calculate fairly accurately the covered distance by physically active elderly. Thus, the equations analyzed in the current study should be used with care by researchers and professionals that deal with this populations in Brazil.

\section{Keywords: motor activity, exercise, aerobic, physical endurance, aging.}

\section{RESUMEN}

Introducción: Debido a la importancia de la prueba de caminata de seis minutos (PC6), algunas ecuaciones han sido propuestas a fin de predecir el resultado esperado. Objetivo: Comparar los valores encontrados en diferentes ecuaciones de referencia para predicción de la distancia en la PC6 en ancianos practicantes de ejercicios físicos. Métodos: Participaron 696 ancianos de ambos sexos, siendo 600 mujeres y 96 hombres, practicantes de ejercicios físicos en el Grupo de Estudios de la Tercera Edad (GETI), el Programa Vivir Activo y el Programa Salud y Ocio. Para evaluar la resistencia aeróbica, se utilizó la PC6, conforme a lo propuesto por Rikli y Jones. Para el cálculo de la distancia recorrida prevista, se utilizaron las ecuaciones de Enright y Sherrill, Troosters y Enright. Se usó estadística descriptiva e inferencial, con nivel de significancia de 5\%. Resultados: Los promedios de las distancias recorridas

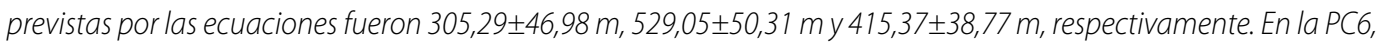


el promedio fue de $513,05 \pm 87,26 \mathrm{~m}$. Se observó correlación significativa $(p<0,001)$ entre la PC6 y las ecuaciones de Enright y Sherrill, y Troosters. En la comparación entre los valores, se nota que las ecuaciones de Enright y Sherrill y Enright subestiman y la de Troosters superestima la resistencia aeróbica de ancianos practicantes de ejercicios físicos. Conclusión: Las ecuaciones de referencia para predicción de la distancia recorrida en la PC6, analizadas en este estudio, no reflejan la resistencia aeróbica de ancianos practicantes de ejercicio físicos. Nuevas ecuaciones y/o variables deben ser incluidas para calcular, con cierta precisión, la distancia recorrida por ancianos físicamente activos. De esa forma, las ecuaciones analizadas en este estudio deben ser utilizadas con cautela por investigadores y profesionales que trabajan con ese público en Brasil.

Palabras clave: actividad física, ejercicio, resistencia física, envejecimiento.

\section{INTRODUÇÃO}

O teste de caminhada de 6 minutos (TC6) vem sendo amplamente utilizado para avaliar a capacidade cardiopulmonar tanto de indivíduos saudáveis quanto de indivíduos com alguma doença instalada'. Trata-se de um teste submáximo, não oneroso, rápido e simples que se correlaciona com o consumo de oxigênio máximo e as atividades de vida diária ${ }^{2-6}$.

O desempenho neste teste tem sido usado como medida da capacidade funcional de pacientes com doenças cardiopulmonares crônicas graves, que não podem realizar provas físicas com esforço máximo. Além de verificar a capacidade aeróbia, recentemente, tem-se reconhecido o TC6 como um preditor da capacidade física global e da mobilidade em idosos e não apenas uma medida específica da capacidade cardiovascular?.

Devido a importância desse teste, algumas equações têm sido propostas para predizer o seu resultado esperado. Na população idosa, as equações de Enright \& Sherrill ${ }^{6}$, Troosters et al. ${ }^{5}$ e Enright et al. ${ }^{4}$ vêm sendo empregadas para avaliar a distância percorrida prevista no TC6. Essas equações consideram como variáveis para o cálculo o sexo, a idade, o peso e a altura.

Alguns estudos nacionais já foram realizados para avaliar a aplicabilidade dessas equações. Soares et al. ${ }^{8}$ estimou a distância a ser percorrida no TC6 por meio da equação de Enright \& Sherrill ${ }^{6}$ em adultos com idade entre 40 e 80 anos e encontrou resultados semelhantes. Na população idosa saudável (idade entre 64 e 82 anos), Barata et al. ${ }^{3}$ encontrou grande variação entre as distâncias percorridas e as previstas pelas equações de Enright \& Sherrill ${ }^{6}$, Troosters et al. ${ }^{5}$ e Enright et al. ${ }^{4}$.

No entanto, ambas as pesquisas supracitadas apresentaram uma amostra relativamente pequena para gerar resultados satisfatórios (38 indivíduos). Além disso, não foram encontrados estudos que avaliassem se essas equações são adequadas para representar a capacidade aeróbica de idosos praticantes de exercícios físicos. Portanto, o objetivo desse estudo é comparar os valores encontrados em diferentes equações de referência para predição da distância percorrida e no teste de caminhada de 6 minutos (resistência aeróbica) em idosos praticantes de exercícios físicos.

\section{MÉTODOS E CASUÍSTICA}

Este estudo foi realizado com os idosos participantes do projeto de exercícios físicos do Grupo de Estudos da Terceira Idade (GETI) da Universidade do Estado de Santa Catarina (UDESC), Programa Viver Ativo da Secretaria de Assistência Social (SAS) da Prefeitura Municipal de Florianópolis/SC e do Programa Saúde e Lazer do Instituto Federal de Santa Catarina (IF/SC). Estes programas atendem uma população de aproximadamente 2000 idosos. A amostra foi selecionada de forma intencional, tendo-se como critérios de inclusão: idade ( $\geq 60$ anos) e praticantes de modalidades de exercícios físicos (ginástica, hidroginástica, natação, caminhada e dança). Dessa forma, fizeram parte do estudo 696 idosos de ambos os sexos, sendo 600 mulheres e 96 homens, que estão inseridos nos respectivos programas há aproximadamente dois anos.

As aulas dos programas têm duração de 50 minutos e são realizadas de duas a três vezes por semana. Essas aulas prezam por uma intensidade moderada em que os idosos precisam de algum esforço físico para realizá-las, bem como a necessidade de impor uma respiração um pouco mais intensa do que o normal.

Para caracterização da amostra, foi utilizada uma ficha diagnóstica contendo informações como idade e sexo. Também foram mensuradas a massa corporal e a estatura dos idosos por meio de uma balança digital Plenna Wind MEA 07710 e um estadiômetro WCS $217 \mathrm{~cm}$ com plataforma da marca CARDIOMED, respectivamente. As unidades de medida foram quilograma $(\mathrm{kg})$ e centímetros (cm).

Para avaliar a resistência aeróbica, foi utilizado o teste de caminhada de 6 minutos, conforme proposto na bateria de testes Senior Fitness Test-SFT9 ${ }^{9}$ que é específica para idosos.

Os dados foram coletados por alunos do CEFID/UDESC previamente treinados. Inicialmente, realizou-se um contato pessoal com os idosos do GETI/UDESC, Viver Ativo/SAS e IF/SC, explicando-se o objetivo da pesquisa, o sigilo da identificação e solicitando sua participação. Em seguida, foi agendada a data, o horário e o local para aplicação dos instrumentos do estudo.

As avaliações aconteceram no local onde eram realizados os exercícios físicos. Primeiramente aplicou-se a ficha diagnóstica em forma de entrevista individual. Em seguida, foram mensuradas as medidas antropométricas e aplicado o teste de caminhada de 6 minutos ${ }^{9}$.

Os dados foram organizados no programa Microsoft Exce ${ }^{\circledR}$ e analisados no programa estatístico SPSS - Statistical Package for Social Sciences - versão 17.0 para Windows.

Para o cálculo da Distância Percorrida Prevista (DPP) de Enright \& Sherrill ${ }^{6}$, Troosters et al. ${ }^{5}$ e Enright et al. ${ }^{4}$, as variáveis massa corporal, altura e idade foram inseridos nas respectivas fórmulas, expressas na tabela 1.

O tratamento estatístico descritivo foi realizado mediante frequência simples, porcentagens, média e desvio padrão. Para análise inferencial, foi utilizado o teste $U$ de Mann Whitney para comparar as características dos sujeitos, segundo o sexo, correlação de Spearman (Rho) para relacionar os valores previstos e o mensurado e o teste de Wilcoxon (W) para comparar esses valores. Adotou-se um nível de significância de 5\%.

Essa pesquisa foi conduzida dentro dos padrões exigidos pela resolução n. 196/96 do Conselho Nacional de Saúde do Brasil e aprovada pelo Comitê de Ética em Pesquisas em Seres Humanos da Universidade do Estado de Santa Catarina (UDESC), sob o protocolo número 185/07.

Anterior à aplicação das avaliações, os idosos que concordaram em participar da pesquisa, assinaram o termo de consentimento em duas vias, uma de posse do idoso e a outra do pesquisador. 
Tabela 1. Equações de referência propostas por autores para cálculo da distância percorrida no teste de caminhada de seis minutos.

\begin{tabular}{c|l}
\hline \multicolumn{1}{c}{ Enright \& Sherrill $^{6}$} \\
\hline Homem & $\begin{array}{l}\text { Distância prevista }=(7,57 \times \text { altura } \mathrm{cm})-(5,02 \times \text { idade })- \\
(1,76 \times \text { peso kg })-309 \mathrm{~m}\end{array}$ \\
\hline Mulher & $\begin{array}{l}\text { Distância prevista }=(2,11 \times \text { altura } \mathrm{cm})-(2,29 \times \text { peso } \mathrm{kg})- \\
(5,78 \times \text { idade })+667 \mathrm{~m}\end{array}$ \\
\hline Homem & $\begin{array}{l}\text { Distância prevista }=218+(5,14 \times \text { altura } \mathrm{cm}-5,32 \times \text { idade })- \\
(1,80 \times \text { peso kg }+51,31)\end{array}$ \\
\hline Mulher & $\begin{array}{l}\text { Distância prevista }=218+(5,14 \times \text { altura } \mathrm{cm}-5,32 \times \text { idade })- \\
(1,80 \times \text { peso kg })\end{array}$ \\
\hline Enright et al. ${ }^{4}$ \\
\hline Homem & $\begin{array}{l}\text { Distância prevista }=493+(2,2 \times \text { altura } \mathrm{cm})-(0,93 \times \text { peso kg })- \\
(5,3 \times \text { idade })+17 \mathrm{~m}\end{array}$ \\
\hline Mulher & $\begin{array}{l}\text { Distância prevista }=493+(2,2 \times \text { altura } \mathrm{cm})-(0,93 \times \text { peso kg })- \\
(5,3 \times \text { idade })\end{array}$ \\
\hline
\end{tabular}

\section{RESULTADOS}

Foram entrevistados 696 idosos praticantes de exercícios físicos, sendo 600 mulheres $(n=86,2 \%)$ e 96 homens $(n=13,8 \%)$. As características dos sujeitos podem ser observadas na tabela 2 .

Nota-se que os homens apresentam maior idade, peso e estatura, quando comparados às mulheres $(p<0,05)$. Além disso, com relação ao teste de caminhada de seis minutos, se observa que a média dos idosos foi de 513,05 $\pm 87,26 \mathrm{~m}$, havendo diferença significativa entre os sexos.

Quanto às equações de referência para predição da distância percorrida, nota-se que a fórmula de Enright \& Sherrill ${ }^{6}$ apresentou média de $305,29 \pm 46,98 \mathrm{~m}$, enquanto de Troosters et al. ${ }^{5}$ foi de 529,05 $\pm 50,31 \mathrm{~m} \mathrm{e}$

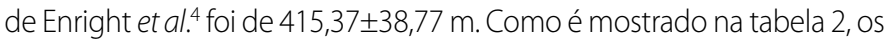
homens apresentaram maiores valores que as mulheres, com diferença significativa nas fórmulas de Troosters et al. ${ }^{6}$ e de Enright et al. ${ }^{4}$.

Quanto à relação entre os valores mensurados (TC6) e aqueles calculados segundo as equações de referência, nota-se que há correlação significativa $(p<0,001)$ com as fórmulas de Enright \& Sherrill ${ }^{6} \mathrm{e}$ Troosters et al. ${ }^{5}$. Ambas as correlações podem ser verificadas na figura 1.

Observa-se que essas correlações são moderadas e que 18,1\% da distância percorrida no TC6 pode ser explicada pela variabilidade da distância percorrida prevista pela equação de Enright \& Sherrill6, enquanto $17,1 \%$ pela equação de Troosters et al. ${ }^{5}$. Não foi encontrada correlação significativa entre a distância percorrida no TC6 e os valores encontrados pela equação de Enright et al. ${ }^{4}$.

Na comparação entre os valores obtidos no TC6 e a distância percorrida prevista pelas equações (figura 2), observa-se que as fórmulas de Enright \& Sherrill ${ }^{6}$ e Enright et al. ${ }^{4}$ subestimam a resistência aeróbica de idosos praticantes de exercícios físicos. A ilustração reitera os dados da Tabela 2, demonstrando que a diferença entre a distância percorrida no TC6 (513,05 \pm 87,26 m) e as equações (305,29 \pm 46,98 e 415,37 \pm $38,77 \mathrm{~m}$, respectivamente), são significativamente inferiores.

No entanto, com relação à distância percorrida prevista pela equação de Troosters et al. ${ }^{5}$, nota-se que esses valores (529,05 \pm 50,31 m) são superiores àqueles obtidos no TC6, sugerindo que essa fórmula superestima a real resistência aeróbica de idosos praticantes de exercícios físicos.

A comparação entre os valores obtidos no TC6 e a distância percorrida prevista pelas equações também foi realizada segundo o sexo dos idosos participantes (figura 3). Assim como observado na amostra total, as fórmulas de Enright \& Sherrill 6 e Enright et al. ${ }^{4}$ subestimam e a fórmula de Troosters et al. ${ }^{5}$ superestima a resistência aeróbica de mulheres idosas praticantes de exercícios físicos. Entre os homens, observou-se que todas as equações de referência subestimam os valores obtidos no teste de caminhada de seis minutos $(p<0,05)$.

Tabela 2. Características dos sujeitos do estudo $(n=696)$.

\begin{tabular}{|c|c|c|c|c|c|}
\hline \multirow[t]{2}{*}{ Características } & $\begin{array}{c}\text { Homens } \\
(n=96)\end{array}$ & $\begin{array}{l}\text { Mulheres } \\
(n=600)\end{array}$ & $\begin{array}{c}\text { Total } \\
(n=696)\end{array}$ & \multirow[t]{2}{*}{$U$} & \multirow[t]{2}{*}{$p$} \\
\hline & Média $( \pm D P)$ & Média ( $\pm D P$ ) & Média ( $\pm \mathrm{DP})$ & & \\
\hline Idade (anos) & $70,05(6,28)$ & $68,21(6,20)$ & $68,47(6,24)$ & 23742,5 & $0,006^{*}$ \\
\hline Peso (kg) & $73,99(11,99)$ & $68,70(12,03)$ & $69,46(12,16)$ & 21881,5 & $<0,0001^{*}$ \\
\hline Altura $(\mathrm{cm})$ & $165,35(8,01)$ & $155,68(6,74)$ & $157,05(157,05)$ & 10226,5 & $<0,0001^{*}$ \\
\hline TC6 & $543,23(90,12)$ & $508,04(85,83)$ & $513,05(87,26)$ & 20569,5 & $<0,0001^{*}$ \\
\hline $\begin{array}{c}\text { Enright \& } \\
\text { Sherrill(1998) }\end{array}$ & $307,80(64,47)$ & $304,88(43,46)$ & $305,29(46,98)$ & 27157,0 & 0,369 \\
\hline $\begin{array}{l}\text { Troosters } \\
\text { et al.(1999) }\end{array}$ & $511,13(51,55)$ & $532,02(49,52)$ & $529,05(50,31)$ & 22412,0 & $0,001^{*}$ \\
\hline $\begin{array}{l}\text { Enright } \\
\text { et al.(2003) }\end{array}$ & $433,25(38,27)$ & $412,41(38,08)$ & $415,37(38,77)$ & 19446,5 & $<0,0001^{*}$ \\
\hline
\end{tabular}

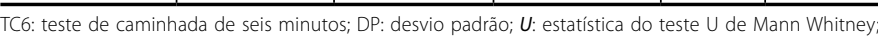
p: nível de significância.
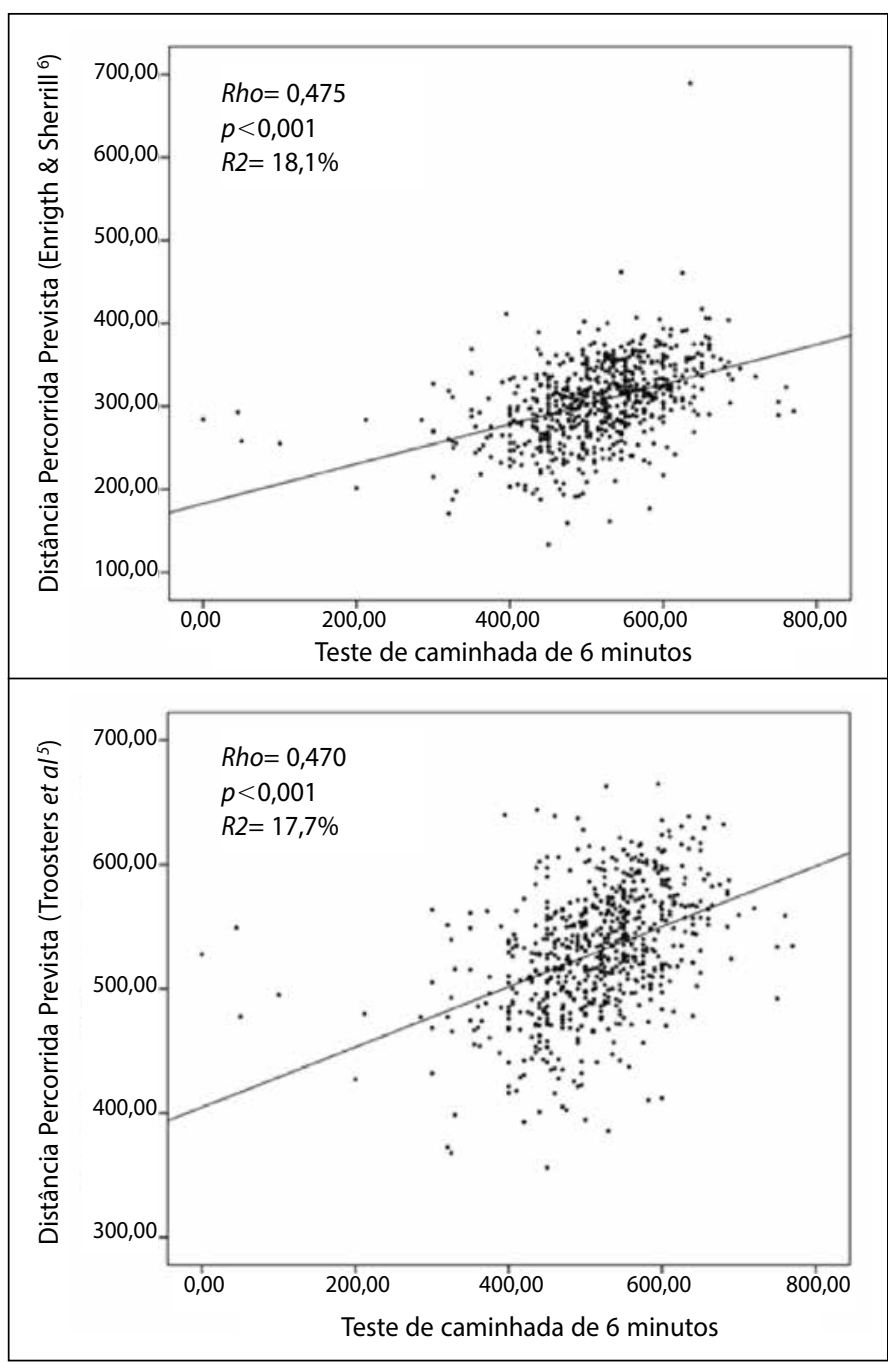

Figura 1. Correlação entre a distância percorrida no teste de caminhada de seis minutos e as distâncias percorridas segundo as equações de Enrigth \& Sherrill ${ }^{6}$ e Troosters et al. ${ }^{5}$. Rho: estatística da correlação de Spearman; p: nível de significância; R2: coeficiente de determinação. 


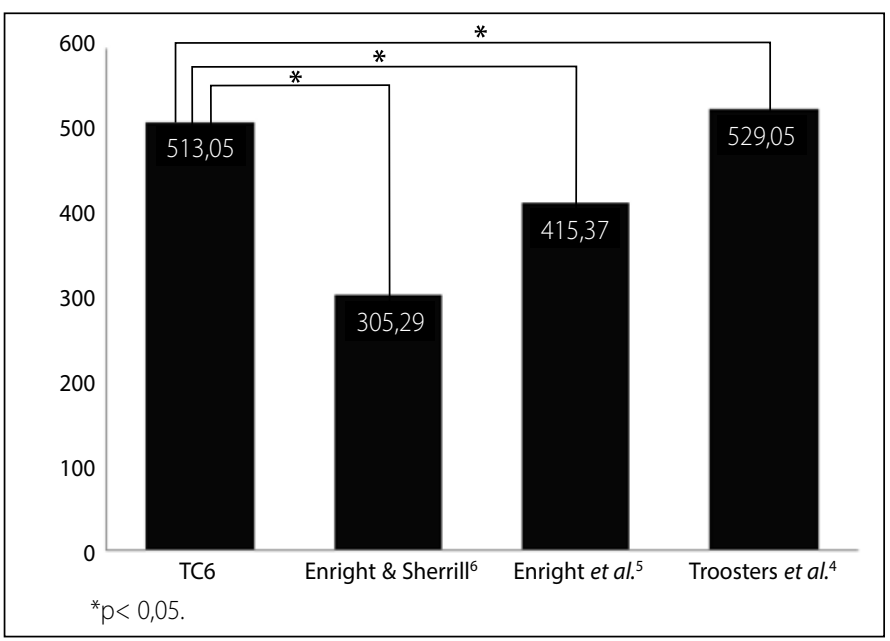

Figura 2. Comparação entre a distância percorrida no teste de caminhada de seis minutos (TC6) e a distâncias percorridas segundo as equações de Enrigth \& Sherrill (1998), Troosters et al. (1999) e Enrigth et al. (2003).

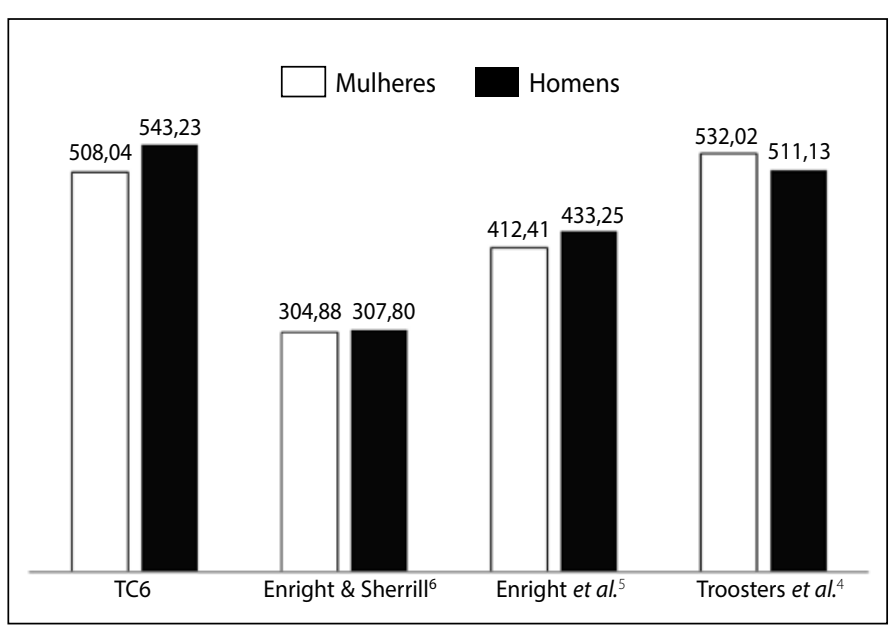

Figura 3. Comparação entre a distância percorrida no teste de caminhada de seis minutos (TC6) e a distâncias percorridas nas equações de Enrigth \& Sherrill6, Troosters et al..$^{5}$ e Enrigth et al. ${ }^{4}$, segundo o sexo.

\section{DISCUSSÃO}

O teste de caminhada, primeiramente proposto por McGavin ${ }^{10}$, foi desenvolvido como ferramenta de avaliação da capacidade funcional de portadores de pneumopatias crônicas. Nessa perspectiva, estudos desenvolveram equações para estimar o resultado do teste de seis minutos. A equação desenvolvida por Enright \& Sherrill ${ }^{6}$ utilizou uma amostra randomizada composta por 117 homens e 173 mulheres com idade de 40 a 80 anos, sendo excluídos indivíduos que fumavam ou apresentavam indicadores de doenças pré-determinadas. Já na fórmula proposta por Enright et al. ${ }^{4}$, a amostra foi por conveniência e contou com a participação de 437 mulheres e 315 homens saudáveis com idade superior ou igual a 68 anos de idade; excluindo-se pessoas com algumas limitações que pudessem influenciar no seu desempenho durante o teste. Quando Troosters et al. ${ }^{5}$ propuseram sua equação, foram avaliados 53 indivíduos belgas saudáveis e sedentários com idades de 50 a 85 anos; não puderam ser testadas pessoas lesionadas ou que possuíam história de hospitalizações ou doenças capazes de afetar a capacidade de caminhar. Contudo, percebe-se que nenhuma das equações analisadas no presente estudo utilizaram apenas indivíduos fisicamente ativos.

Ainda que seja muito importante para profissionais e pesquisadores obter parâmetros para indivíduos idosos e fisicamente ativos, obser- vam-se algumas limitações no estudo como: ausência de avaliadores cegos e controle de algumas variáveis como tempo de atividade física, modalidade praticada e frequência semanal de prática. Além disso, ainda que o número amostral seja consideravelmente alto, comparado a outros estudos da área, não foi realizado um cálculo amostral.

O presente estudo demonstrou que as fórmulas de Enright \& Sherrill ${ }^{6}$ e Enright et al. ${ }^{4}$ subestimam a resistência aeróbica de idosos praticantes de exercícios físicos, enquanto a fórmula de Troosters et al. ${ }^{5}$ a superestima. Este padrão também foi observado entre mulheres idosas. Já entre os homens, todas as equações subestimam a resistência aeróbica; comportamento inverso ao apresentado pelos sujeitos do estudo de Barata et al. ${ }^{3}$, que avaliaram 38 idosos saudáveis com idades variando de 64 a 82 anos, no qual as três fórmulas analisadas superestimaram a distância percorrida tanto por homens quanto por mulheres.

Os achados do presente estudo apontam que apenas a equação proposta por Troosters et al. ${ }^{5}$ foi semelhante ao encontrado por Barata et al. ${ }^{3}$. Mesmo diante de uma correlação positiva e significativa com a distância percorrida (Figura 1), nota-se que essa equação não subestimou a distância percorrida no TC6 pelos participantes do presente estudo; portanto, observa-se que os idosos atingiram 96,97\% dos valores previstos, apesar da diferença significativa entre esses valores demonstrar que o teste superestima os valores mensurados.

Sabe-se que a distância percorrida no TC6 por idosos sedentários é inferior àqueles ativos fisicamente. No estudo de Pires et a ${ }^{2}$, a distância percorrida média por idosos sedentários foi de 457,39 m. Esse valor é inferior ao encontrado no presente estudo (513,05 \pm 87,26 m). Estes valores superiores são explicados por Silva e Rabello ${ }^{11}$, que apontam que a realização de programas de atividades físicas para a terceira idade promove maior funcionalidade dos sistemas cardiovascular e ósseo.

Segundo Justo e Santos ${ }^{1}$, a equação de Enright e Sherrill ${ }^{6}$ é considerada a mais utilizada para a população idosa. Esses mesmos autores encontraram diferença significativa entre a distância prevista $(448,9 \pm$ 52,7 m) e a distância percorrida no TC6 (491,3 \pm 63,4 m) em 32 idosos com média de idade de 70,8 anos. Apesar da amostra reduzida, os resultados já apontavam que esta equação de referência subestima a capacidade aeróbia da população idosa.

Além disso, outro estudo aponta que a equação proposta por Troosters et al. ${ }^{5}$ também superestimou a "real" distância percorrida por 35 indivíduos saudáveis e com média de idade de $61 \pm 8,3$ anos. Os autores concluem que a equação, derivada da população caucasiana, não prediz a distância percorrida por adultos saudáveis de Cingapura' ${ }^{12}$; tal conclusão também é aplicada para australianos ${ }^{13}$, árabes ${ }^{14}$ e tunisianos $^{15}$, uma vez que a equação de Troosters et al. ${ }^{5}$ indicou valores significativamente diferentes daqueles apresentados no TC6 para estas populações.

A evidente diversidade populacional utilizada para elaboração das equações de referência, associada aos diferentes protocolos do TC6, explica em grande parte a variabilidade dos resultados encontrados nos diversos estudos.

Conforme Barata et al. ${ }^{3}$, existe grande variação entre as distâncias percorridas por idosos brasileiros e as previstas pelas equações, portanto, torna-se necessária a realização de estudos adicionais para confirmar a aplicabilidade dessas equações para a população idosa brasileira. Dourado ${ }^{16}$ reitera essa necessidade ao declarar a importância de valores de referência específicos para cada etnia, população e condições clínicas.

\section{CONCLUSÃO}

Diante dos resultados expostos, observa-se que as equações de referência propostas por Enright \& Sherrill ${ }^{6}$ e Enright et al. ${ }^{4}$ subestimam a capacidade aeróbica de idosos praticantes de exercícios físicos, 
uma vez que estes apresentaram maior distância percorrida no TC6 do que a distância prevista. Com relação à equação de Troosters et al. ${ }^{5}$, observou-se um comportamento inverso, em que os resultados obtidos superestimaram a distância percorrida pelos idosos. O mesmo padrão também foi observado entre mulheres idosas. Por outro lado, entre os homens, todas as equações subestimam a resistência aeróbica. Apesar dessas diferenças significativas, na amostra total e segundo o sexo, as equações de Enright \& Sherrill ${ }^{6}$ e Troosters et al. ${ }^{5}$ correlacionaram-se positiva e moderadamente com os valores da distância percorrida no TC6.
Esses achados demonstram que novas equações e/ou novas variáveis devem ser incluídas para calcular com maior precisão a distância percorrida por idosos fisicamente ativos. Assim sendo, as equações analisadas no presente estudo devem ser utilizadas com cautela por pesquisadores e profissionais que lidam com esta população.

Todos os autores declararam não haver qualquer potencial conflito de interesses referente a este artigo.

\section{REFERÊNCIAS}

1. Justo MR, Santos MGF. Comparação da distância percorrida por idosos saudáveis no teste de caminhada de 6 minutos com as distâncias previstas pelas equações de referência. Anuário da produção de iniciação científica discente. 2009;7:49-55.

2. Pires SR, Oliveira AC, Parreira VF, Britto RR. Teste de caminhada de seis minutos em diferentes faixas etárias e índice de massa corporal. . Rev. Bras. Fisioter. 2007;11:147-51.

3. Barata VF, Gastaldi AC, Mayer AF, Sologuren MJJ. Avaliação das equações de referência para predição da distância percorrida no teste de caminhada de seis minutos em idosos saudáveis brasileiros. Rev. Bras. Fisioter. 2005;9:165-71.

4. Enright PL, McBurnie MA, Bittner V, Tracy, RP, McNamara, R, Arnold A, et al. The 6-min walk test - a quick measure of functional status in elderly subjects. Chest. 2003;123:387-98.

5. Troosters T, Goosselink R, Decramer M. Six minute walking distance in elderly subjects. Eur Respir J.1999;14:270-74

6. Enright PL, Sherril DL. Reference equations for the six-minute walk in healthy adults. J Respir Crit Care Med. 1998;158:1384-87.

7. Lord RS, Menz HB. Psychologic, and health predictors of 6-minute walk performance in older people. Phys Med Rehabil. 2002;83:907-11

8. Soares CPS, Pires SR, Britto RR, Parreira VF. Avaliação da aplicabilidade da equação de referência para estimativa de desempenho no teste de caminhada de 6 minutos em indivíduos saudáveis brasileiros.
Ver. Soc. Cardiol. Estado de São Paulo. 2004:14:1-8.

9. Rikli RE, Jones JC. Teste de Aptidão Física para Idosos. (Tradução de Sonia Regina de Castro Bidutte) Manole, São Paulo. 2008

10. McGavin CR, Grupta SP, McHardy GJ. Twelve-minute walking test for assessing disability in chronic bronchitis. BMJ 1976;1:822-3.

11. Silva M, Rabello HT. Estudo comparativo dos níveis de flexibilidade entre mulheres idosas praticantes de atividade física e não praticantes. Rev Dig Edu Fis. 2006;3:1-15.

12. Poh $\mathrm{H}$, et al. Six minute walk distance in healthy singaporean adults cannot be predicted using reference equations derived from caucasian populations. Respirology. 2006;11:211-16.

13. Camarri B, et al. Six minute walk distance in healthy subjects aged 55-75 years. Respir. Med. 2006;100:658-65

14. Almeri H, Al-Majed S, Al-Howaikan A. Six-min walk test in a healthy adult arab population. Respir. Med. 2009;106:1041-46.

15. Saad HB, et al. 6-Minute walk distance in healthly north africans older than 40 years: influence of parity. Respir. Med. 2009;103:74-84

16. Dourado VZ, Vidotto MC, Guerra RL. Equações de referencia para os testes de caminhada de campo em adultos saudáveis. J. Bras. Pneumol. 2011;37:607-14. 